\title{
Comportement dynamique d'une turbine Francis à charge partielle Comparaison modèle-prototype
}

\author{
A. Fritsch \\ D. Maria \\ EDF-DTG, Grenoble \\ Société Neyrpic, Grenoble
}

Lors du fonctionnement à charge partielle d'une turbine Francis, il apparâ̂t sous la roue une torche de cavitation hélicoïdale qui peut provoquer des perturbations gênantes pour l'exploitant (fluctuations de pression, de puissance...). Après une description générale de ce phénomène, on présente les résultats de mesures effectuées sur un modèle réduit de turbine ainsi que sur un prototype industriel en similitude. On tente ensuite de comparer ces résultats en s'aidant d'un modèle mathématique qui permet de tenir compte des différences existant entre les conduits des deux installations.

\section{Introduction}

Le fonctionnement d'une turbine Francis (fig. 1) à charges partielles s'effectue toujours dans des conditions d'écoulement instationnaire dues à la présence d'un champ tournant en sortie de roue. Ce champ tournant est bien connu et l'on sait qu'il donne naissance à une torche hélicoïdale de cavitation.

Les oscillations de pression induites par ce champ tournant peuvent être amplifiées hydrauliquement (résonance hydraulique dépendant du système: circuit amont/turbine/torche/circuit aval) ou électriquement (résonance électrique) et peuvent ainsi provoquer des perturbations génantes pour l'exploitant (bruit, oscillations de puissance, sollicitations alternées de l'aspirateur, du pivot...).

La réponse du système hydraulique à une excitation forcée induite par le champ tournant est étudiée d'une manière générale et appliquée à un cas réel (modèle et prototype $n_{q} 48$ en similitude), dont nous présentons également les résultats de mesures comparatives.

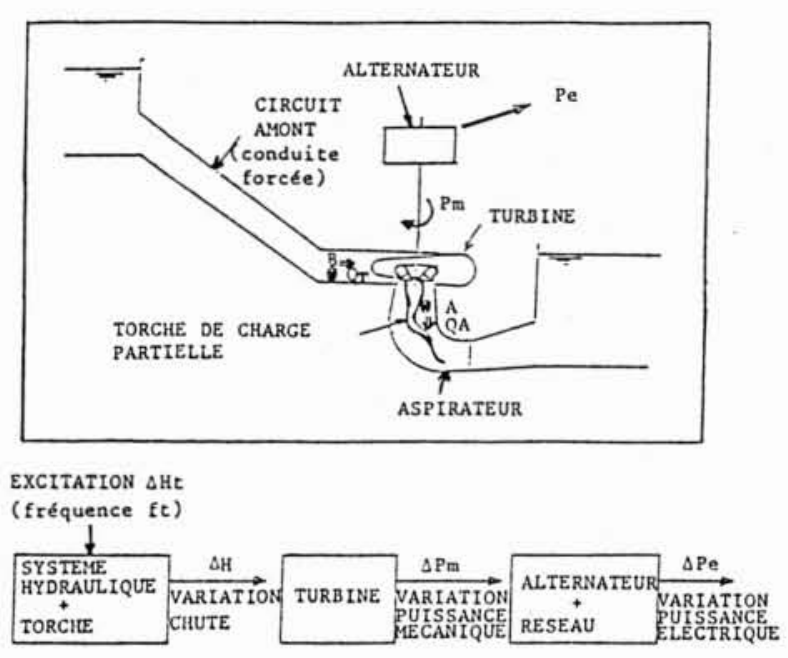

1. Schéma type d'une installation.

Dynamic behaviour of a partial load Francis water turbine. Model/prototype comparison.

During the partial load operation of a Francis water turbine, a helicoidal cavitation torch appears under the wheel and this may provoke interference affecting the operator (pressure fluctuations, power fluctuations, etc). Following a general description of this phenomenon, the results are given of measurements carried out on a turbine reduced model and on a similar industrial prototype. An attempt is then made to compare these results with the help of a mathematical model which takes account of the differences existing between the conduits of the two installations. 


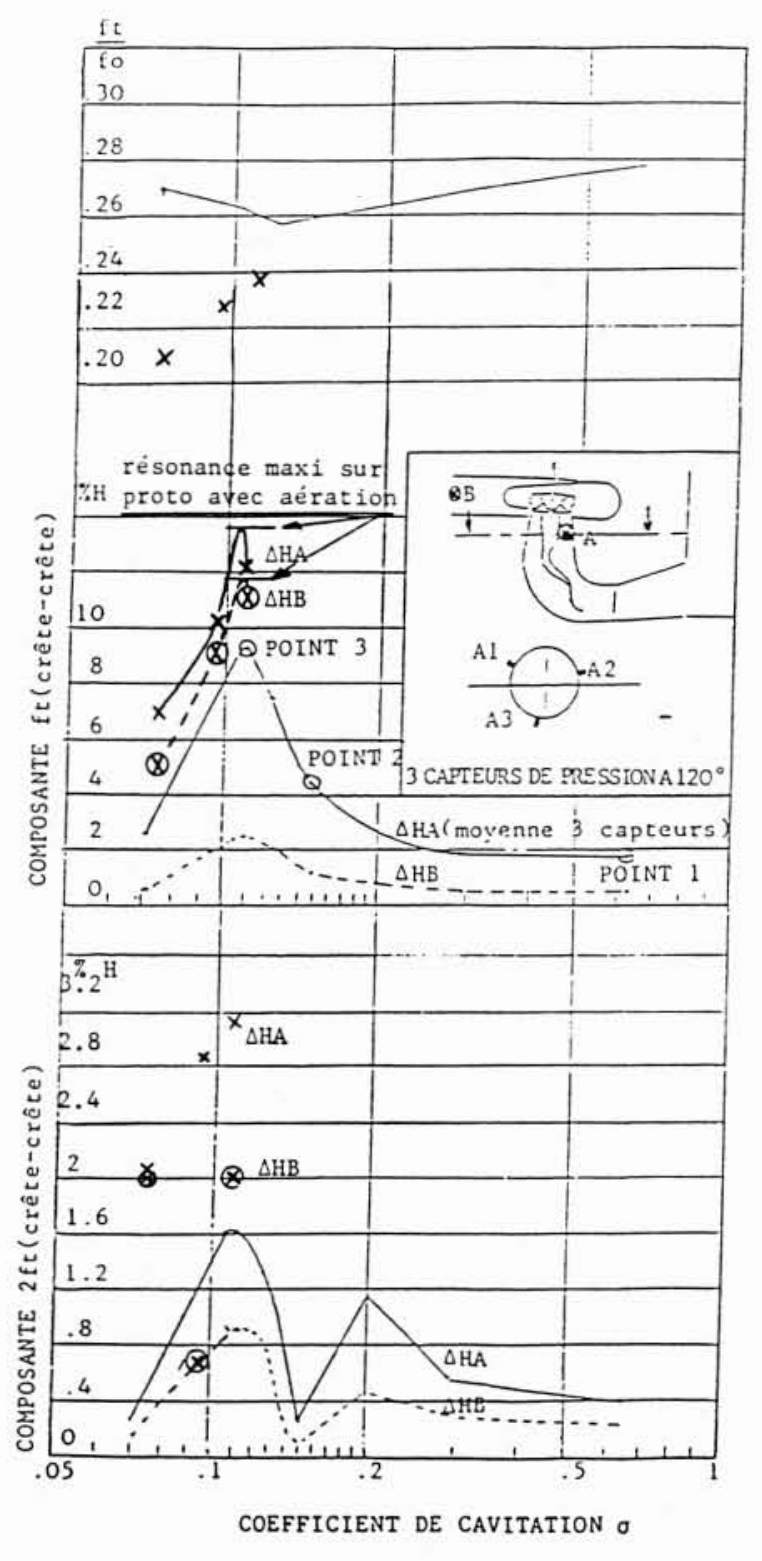

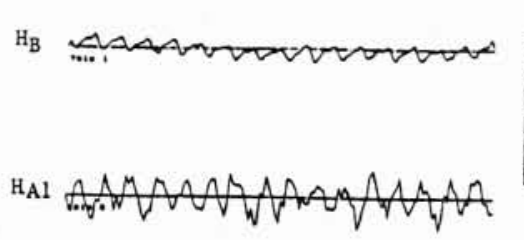
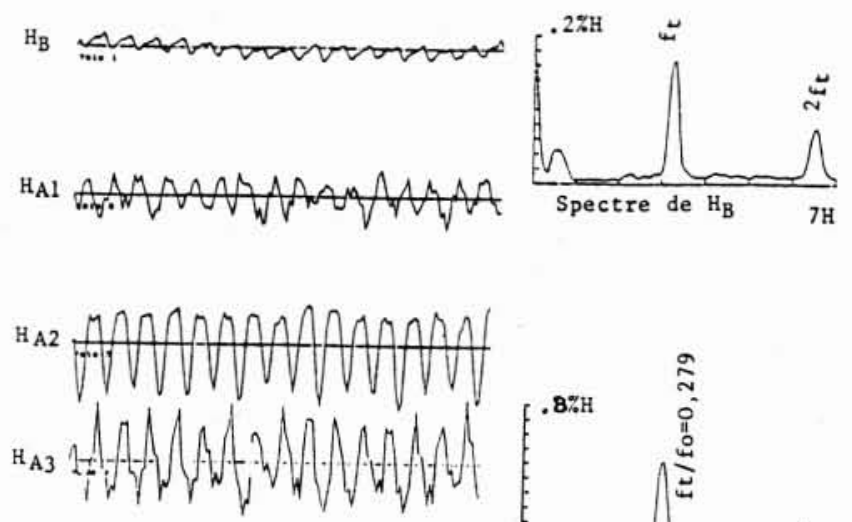

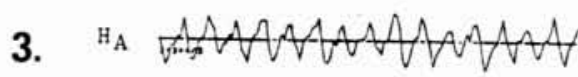

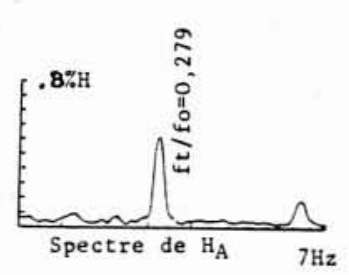

2. Amplitude et fréquence des oscillations de pression à charge partielle sur prototype $n_{q} 48(X)$ et sur modèle en similitude.

3. Modèle $n_{q} 48$ fonctionnant au point 1 de la Figure 2.

4. Modèle $n_{q} 48$ fonctionnant au point 2 de la Figure 2.

5. Modèle $n_{q} 48$ fonctionnant au point 3 de la Figure 2.

Le cas des auto-oscillations en présence d'une torche axiale de forte charge, qui implique nécessairement une théorie non linéaire, n’est pas considéré ici.

\section{Comportement dynamique d'une tur- bine Francis en présence d'une torche de mi-charge}

\section{II.1. Oscillations de pression. Résonance hy- draulique}

La torche hélicoïdale de cavitation (vortex visible en phase vapeur) tourne environ au $1 / 4$ de la vitesse de rotation de la roue. Cette rotation fait apparaitre dans l'aspirateur une excitation $\Delta H_{t}$ due à la déformation du champ tournant dans l'écoulement inhomogène du coude : [2] et [4] ont mis en évidence expérimentalement qu'avec un aspirateur droit, le champ tournant subsiste tandis que l'excitation synchrone disparaît.

L'excitation $\Delta H_{t}$ ne peut être mesurée directement et s'évalue en tenant compte de ses conséquences. L'oscillation synchrone $\Delta H_{A}$ induite dans l'aspirateur peut être déterminée en calculant la valeur moyenne de la pression en plusieurs points d'une même section.

Dans un aspirateur coudé normal, le spectre de l'excitation $\Delta H_{t}$ hors cavitation (sans phase vapeur) a une composante à la fréquence d'excitation $f_{t}$ du champ tournant d'amplitude crête-à-crête variant généralement de 1 à $4 \%$ de la chute, ainsi que des composantes à $2 f_{t}$, $3 f_{t} \ldots$ mais d'amplitudes moindres.

Quand la pression moyenne dans l'aspirateur, caractérisée par le coefficient de cavitation $\sigma$ décroît, le 

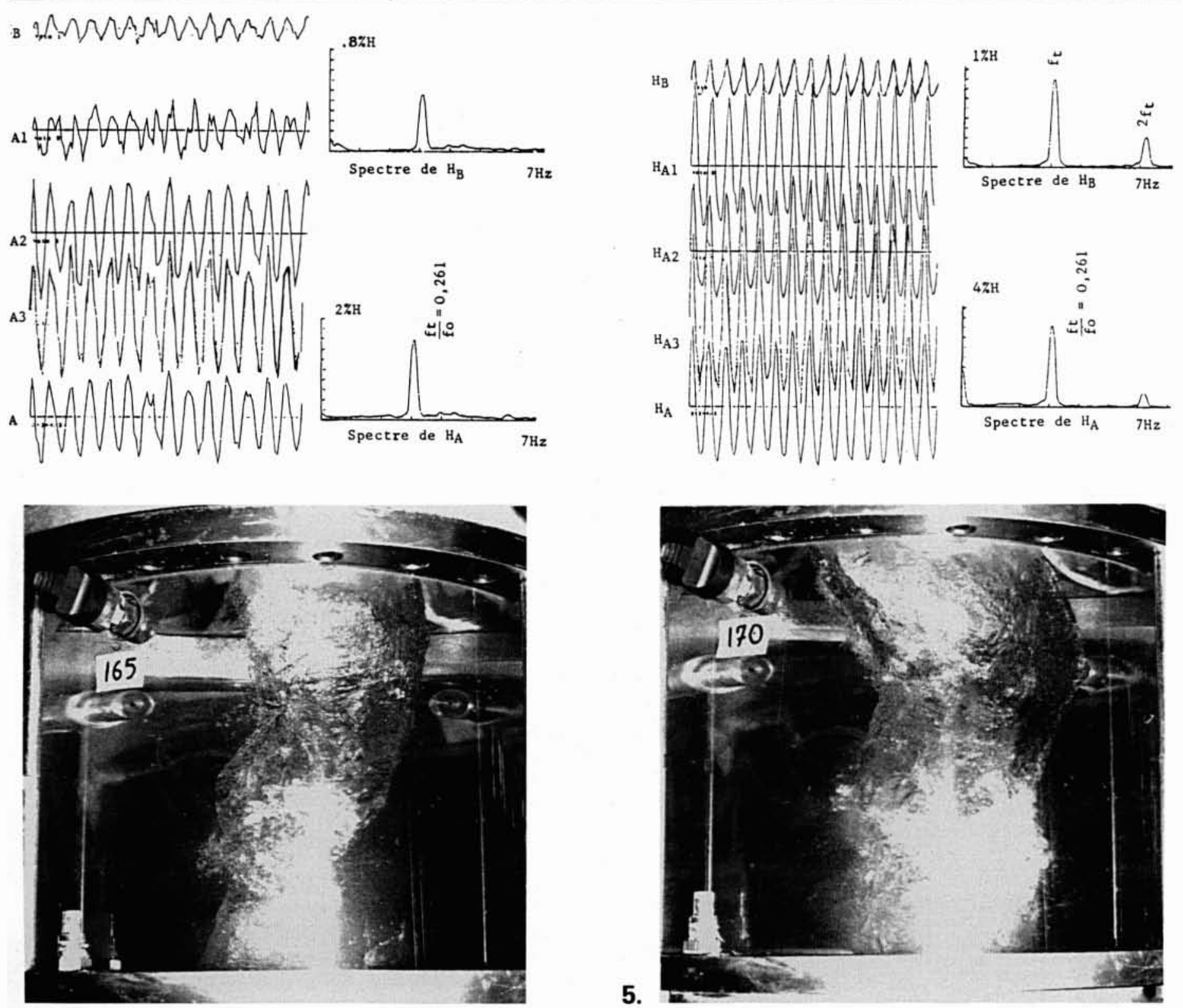

5.

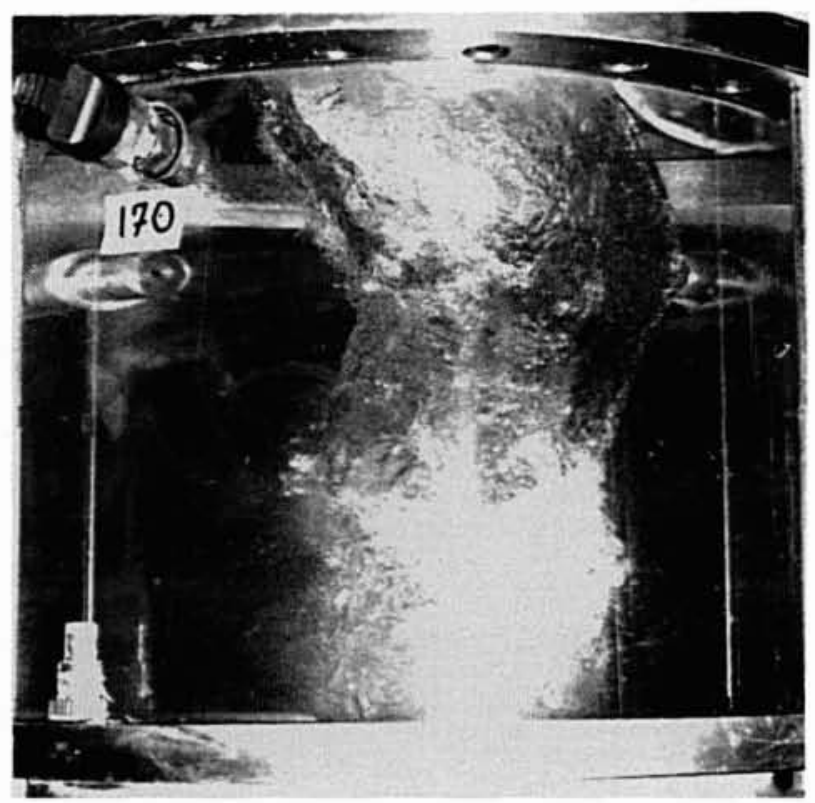

volume de la torche de cavitation augmente et l'oscillation de pression croît jusqu'à une valeur maximale obtenue pour $\sigma_{r m}$ (résonance maximale). Si $\sigma$ continue à décroître, la cavitation augmente, la torche devient quasiment axiale, le champ tournant et donc les oscillations disparaissent (fig. 2 ainsi que réf. [2], [3], [4] et [5]).

Sur la courbe de la figure 2 (amplitude en fonction du $\sigma)$ obtenue sur le modèle $n_{q} 48$ expérimenté, on a repéré trois points :

Point 1: hors cavitation; faible amplitude.

La présence du champ tournant à la fréquence de rotation $f_{t}$ est mis en évidence par les capteurs de pression dans l'aspirateur (fig. 3).

Point 2: Résonance partielle; amplitude croissante.

Les capteurs de pression montrent une superposition du champ tournant et de la pulsation synchrone $\Delta H_{A}$ qui apparait également dans la bâche spirale $\left(\Delta H_{B}\right)$ (fig. 4). Point 3: Résonance maximale.

La pulsation synchrone $\Delta H_{A}$ prédomine (fig. 5).

\section{II.2. Oscillations de puissance - Résonance électrique}

Les oscillations de pression dans l'aspirateur $\Delta H_{A}$, quand elles ne sont pas compensées par des oscillations d'amplitude équivalente dans la bâche spirale $\Delta H_{B}$, induisent des oscillations de chute $\Delta H$ et donc du couple sur l'arbre. Dans le cas d'un alternateur connecté à un réseau, il en résulte donc une oscillation de puissance mécanique $\Delta P_{m}$ qui peut être amplifiée par la machine électrique si la fréquence propre d'oscillation électromagnétique est voisine de la fréquence d'excitation; selon 


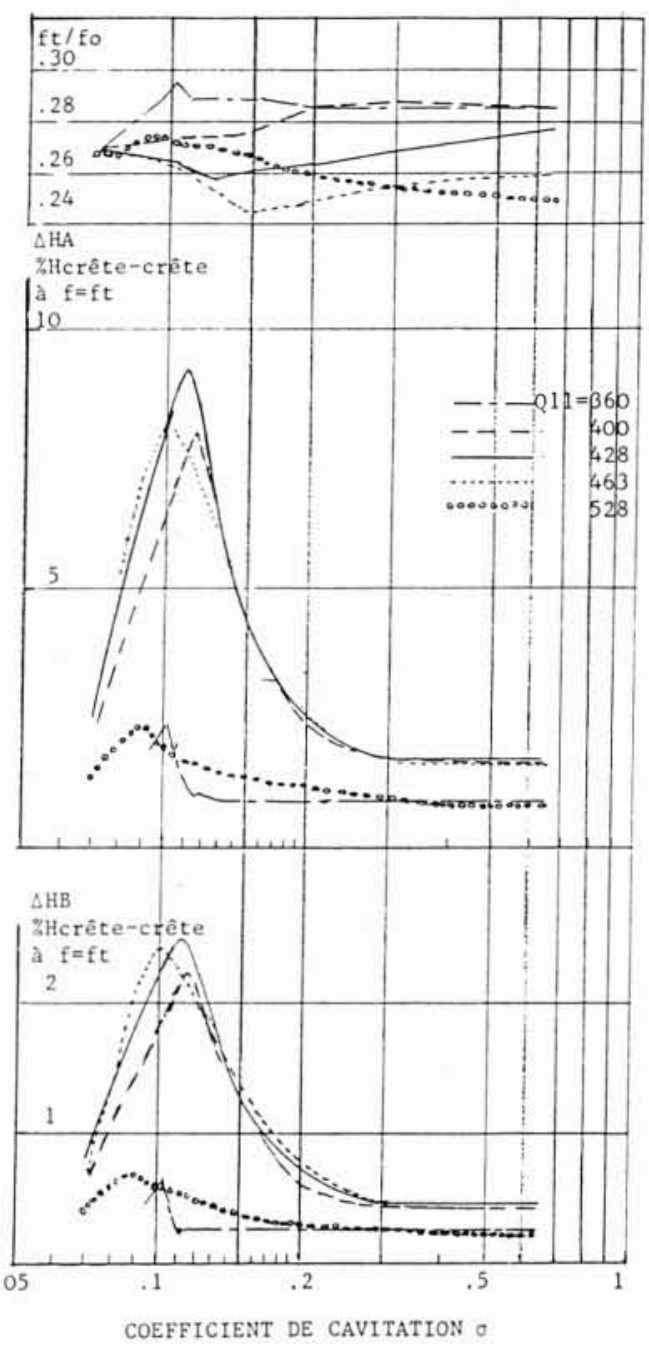

6. Ensemble des résultats d'amplitude et de fréquence des oscillations de pression à charge partielle sur modèle $n_{q} 48$.

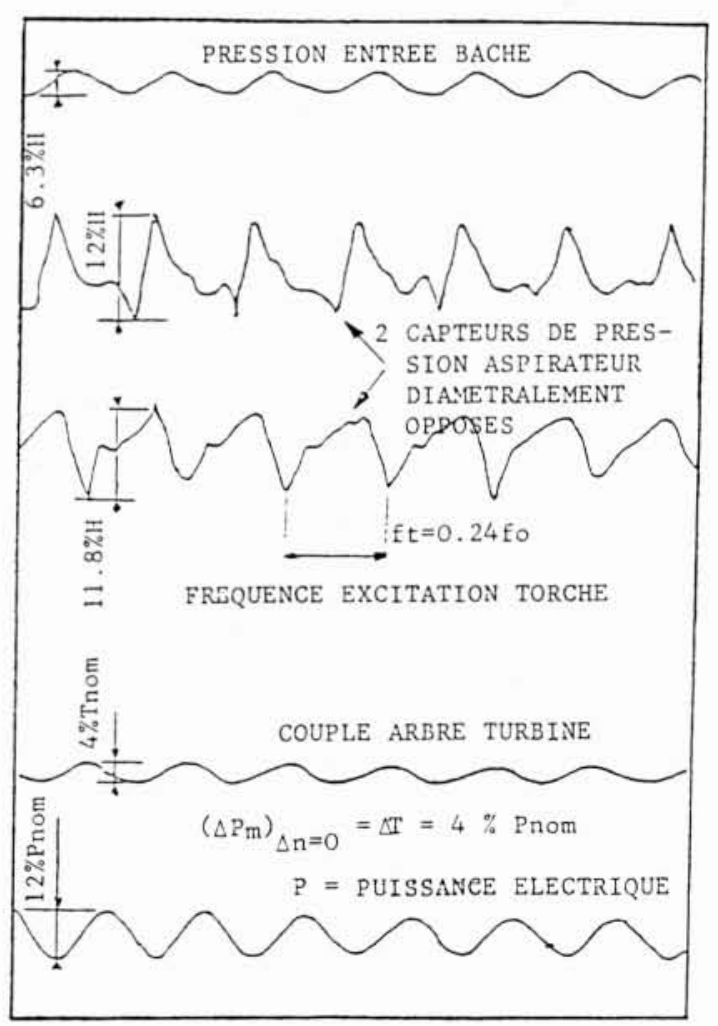

7. Turbine Francis prototype $n_{q} 90$. Fonctionnement à mi-charge avec résonance électrique.

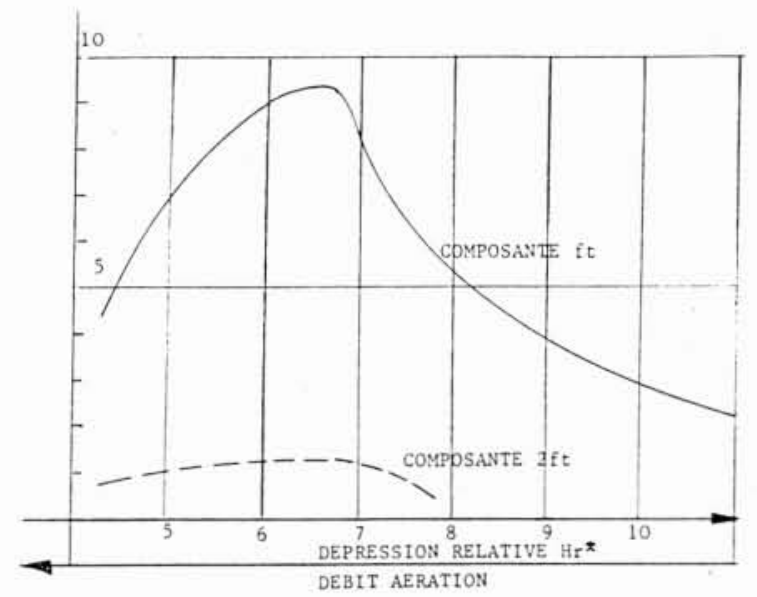

8. Turbine Francis modèle $n_{q} 48$. Influence de l'aération sur les oscillations de pression dans l'aspirateur aux fréquences d'excitation de la torche de mi-charge.

l'importance et le type de réseau, cela peut s'accompagner également d'oscillations de la fréquence électrique.

Les oscillations de puissance mécanique $\Delta P_{m}$ ne représentent généralement que quelques pourcents de la puissance, par contre, on a déjà rencontré des oscillations de puissance électrique $\Delta P e$ supérieures à $10 \%$, du fait d'une amplification importante par l'alternateur. La figure 7 donne un tel exemple d'une turbine Francis $n_{q}$ 90 fonctionnant à mi-charge. La fréquence d'excitation de la torche $f_{t}$ est très proche de la fréquence propre électro-magnétique et il en résulte une amplification $\triangle P e / \Delta P m$ de l'ordre de 3 .

\section{II.3. Influence de l'aération}

L'aération de la torche est un des moyens utilisés pour réduire, quand cela est nécessaire, les perturbations générées par le champ tournant.

Le volume de la torche augmente avec le débit d'air et l'aération a donc un effet similaire à une baisse de $\sigma$. La courbe - amplitude des oscillations de pression en fonction du $\sigma-$ obtenue sans aération (figure 2), donne donc une idée des conséquences d'un débit d'aération croissant. Ainsi, quand le $\sigma$ de l'installation est supérieur à $\sigma_{r m}$ l'aération commence par provoquer un accroissement des oscillations de pression et il est difficile de prévoir le résultat obtenu avec le débit d'air maximum admis naturellement: dans certains cas, les oscillations seront suffisamment atténuées, voire éliminées (torche devenue axiale), mais dans d'autres cas, l'effet de l'aération aura été négatif. Pratiquement, c'est donc lors de la mise en route d'une turbine industrielle que l'on peut optimiser l'aération.

La figure 8 montre l'effet de l'aération obtenu sur le modèle $n_{q} 48$. Partant d'une résonance partielle à $\sigma=0,17$, on voit que l'amplitude des oscillations de pression augmente quand la dépression relative sous la pointe de roue $H_{r}^{*}$ diminue (autrement dit, quand le débit d'aération augmente), passe par un maximum correspondant à celui du $\sigma_{r m}$ sans aération, puis diminue jusqu'à ce que la torche devenue quasiment axiale élimine l'excitation du champ tournant. 


\section{Modèle mathématique linéaire}

\section{III.1. Formulation}

L'analyse théorique de ces oscillations est possible en utilisant les caractéristiques dynamiques linéaires de chaque composant du système (voir fig. 1). Le schéma fonctionnel du modèle utilisé est le suivant:

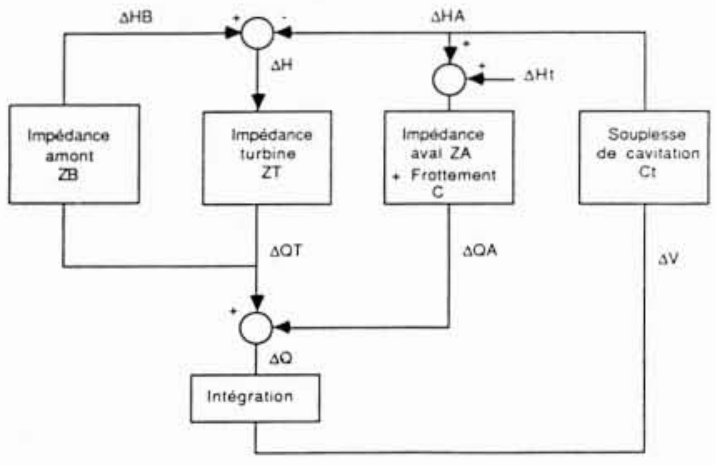

il permet de calculer la réponse du système à l'excitation synchrone : $h_{t}=\Delta H_{t} e^{\text {kot }}$ produite par le champ tournant dans l'aspirateur.

Les principales hypothèses sont les suivantes:

- la souplesse de cavitation $C_{t}$ (Cavitation compliance) est supposée être localisée au point $A$, en sortie de roue. - l'effet de la variation du débit turbiné $\Delta Q_{T}$ sur le volume $\Delta V$ est négligeable.

- l'impédance de la turbine $Z_{T}$ s'écrit: $Z_{T}=R_{T}+j I_{T}$ avec :

$R_{T}=$ partie réelle définie par la tangente sur l'isouverture $=(\Delta H) /(\Delta Q)_{0, n}$

$I_{T}=$ partie imaginaire correspond à l'impédance d'un tronçon de conduite (la bâche spirale principalement). Les résultats ont été vérifiés expérimentalement sur un modèle à partir de la fonction de transfert $\Delta H_{B} / \Delta H_{A}[11]$. - l'impédance d'un tronçon de conduite s'écrit: $Z=j Z_{0} \operatorname{tg}(\omega L / a)$

Dans le cas d'un circuit complexe, $Z_{A}$ et $Z_{B}$ peuvent être déterminés par la méthode des impédances ou des matrices de transfert ([6], [7], [8], [9]).

Ce modèle mathématique nous conduit donc à l'expression complexe de la réponse du système à une excitation $\Delta H_{t}$ à la fréquence $f=f_{t}=\omega / 2 \pi$ et qui s'écrit, hors amortissement :

$$
\Delta H_{A} / \Delta H_{t}=\frac{1}{1+Z_{A}\left(\mathrm{j} \omega C_{t}+1 /\left(Z_{T}-Z_{B}\right)\right)}
$$

Les fréquences de résonance sont les valeurs de $f$ qui rendent maximum la fonction $\Delta H_{A} / \Delta H_{t}$.

\section{III.2. Résultats}

\section{Influence de la fréquence d'excitation}

Les courbes de réponse $\Delta H_{A} / \Delta H_{t}$, calculées en fonction de la fréquence d'excitation $f$, sont données en figure 9 pour différentes valeurs de la souplesse de cavitation $C_{t}$.

\section{Influence de l'amont}

Les courbes de réponse $\Delta H_{A} / \Delta H t$, calculées en fonction de $K_{i}^{*}$ pour la fréquence d'excitation de la torche $f_{t}$, sont données sur la figure 10 pour différentes valeurs de la fréquence propre amont. On observe que la résonance maximale se produit à souplesse de cavitation pratiquement inchangée; par contre, l'amplitude varie et devient maximum quand $f_{\text {pam }}=f_{t}$. Ce résultat a été expérimentalement mis en évidence hors cavitation par [10].

La forme de ce type de courbe correspond bien à celle que nous obtenons expérimentalement en fonction du paramètre $\sigma$.

\section{Influence de l'aval}

Les courbes de réponse $\Delta H_{A} / \Delta H_{t}$ calculées en fonction de $K_{t}$ sont données en figure 11 pour différentes valeurs de la longueur du conduit aval. Quand celle-ci augmente, la résonance se produit à souplesse de cavitation plus faible. A souplesse constante, la fréquence propre du système dépend fortement de l'impédance de l'aval.

9. Courbe de réponse théorique en fonction de la fréquence d'oscillation pour différentes valeurs de la souplesse de cavitation dans le cas du prototype $n_{q} 48$.

10. Courbe de rêponse théorique en fonction de la souplesse inverse de cavitation pour différentes valeurs de la fréquence propre amont dans le cas du prototype $n_{q} 48$.

11. Courbe de réponse théorique en fonction de la souplesse inverse de cavitation pour différentes valeurs de la longueur de l'aspirateur dans le cas du prototype $n_{q} 48$.

9.

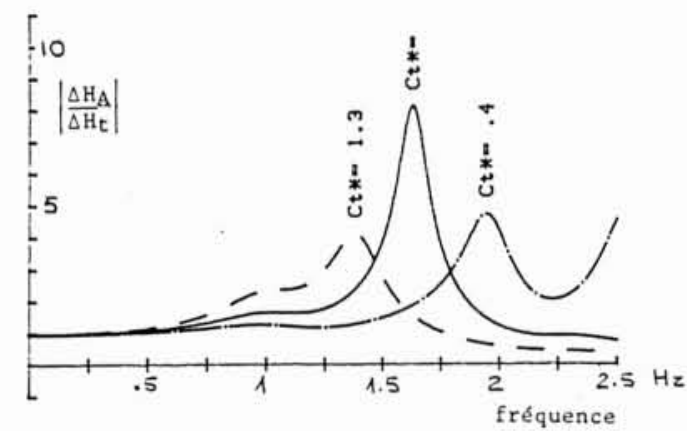

10.

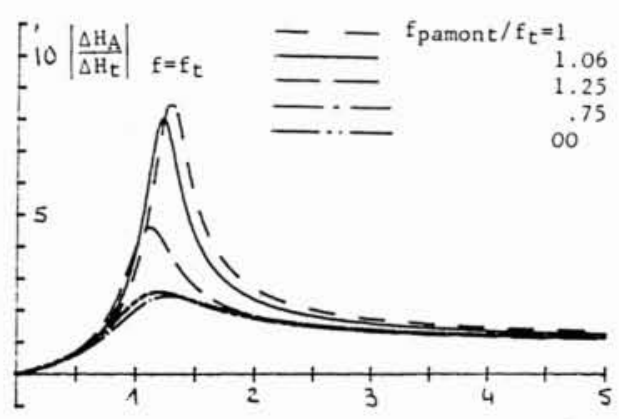

$k_{t}{ }^{*}$

11.

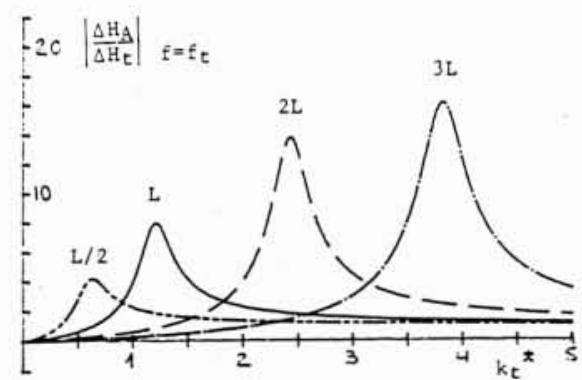



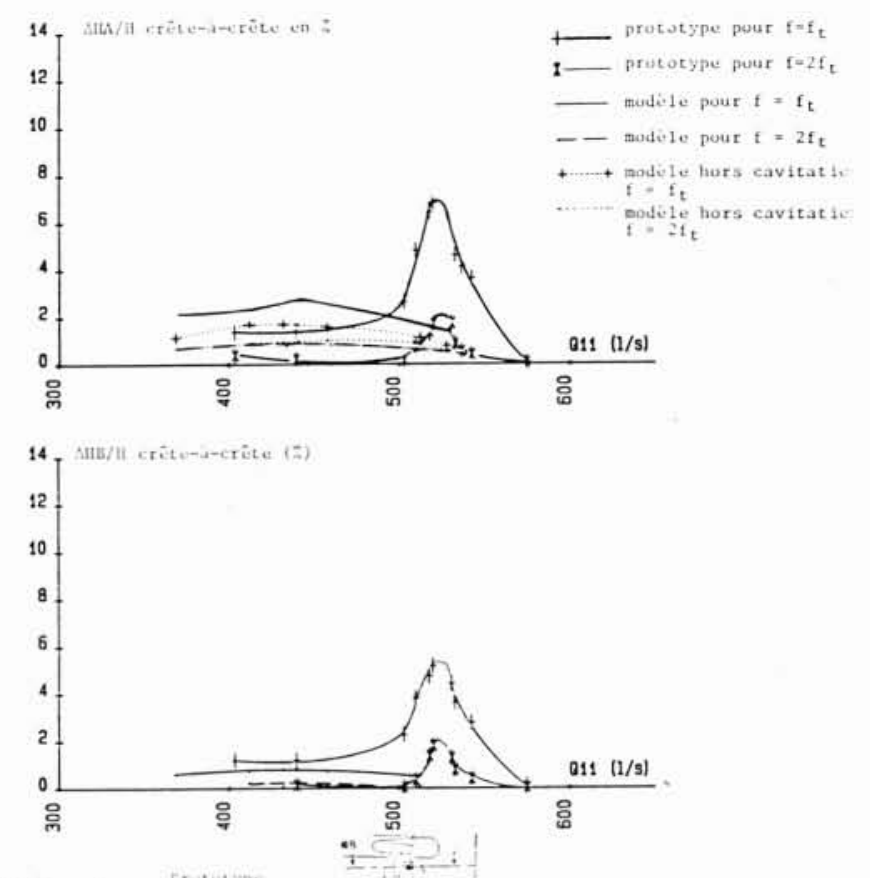

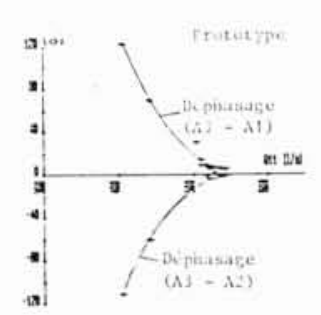

12.
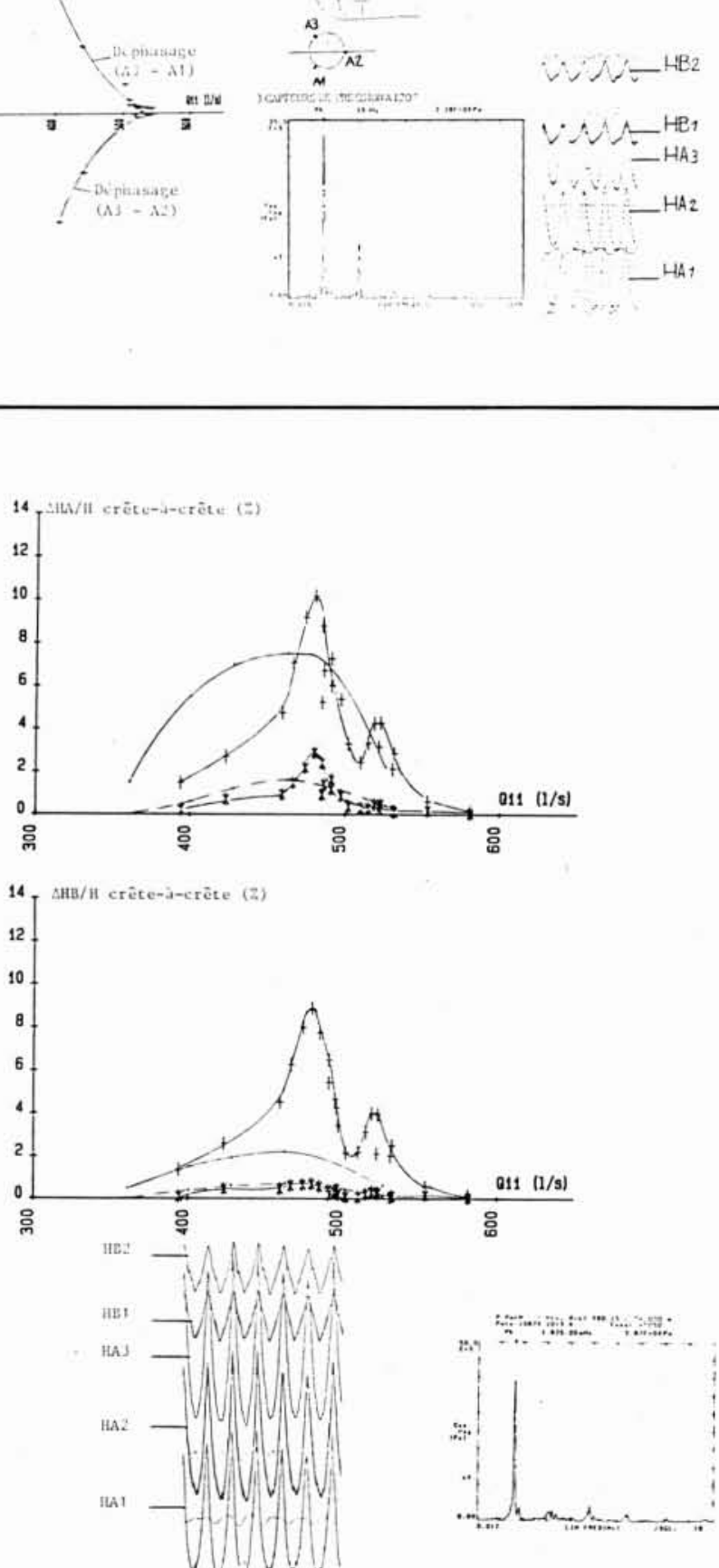
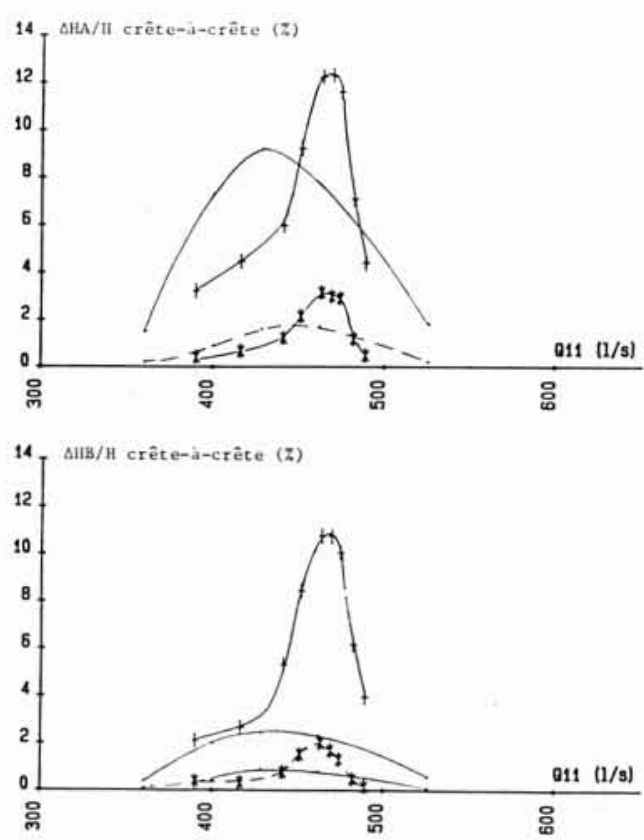

14.

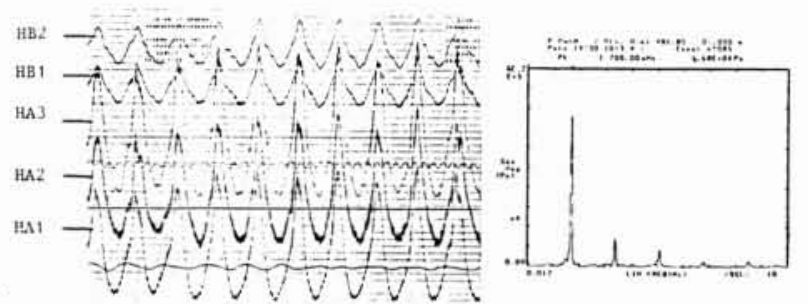

12. Résultats obtenus sur prototype $n_{q} 48$ et sur modèle en similitude avec $\sigma=0.074$

13. Résultats obtenus sur prototype $n_{q} 48$ et sur modèle en similitude avec $\sigma=0.095$.

14. Résultats obtenus sur prototype $n_{q} 48$ et sur modèle en similitude avec $\sigma=0.109$.

15. Courbe de réponse théorique en fonction de la souplesse inverse de cavitation pour le modèle et le prototype $n_{q} 48$.

16. Courbe de réponse expérimentale en fonction du paramètre de cavitation pour modèle $n_{q} 84$. Comparaison avec prototype pour $\sigma$ éloigné de $\sigma_{m}$

15.
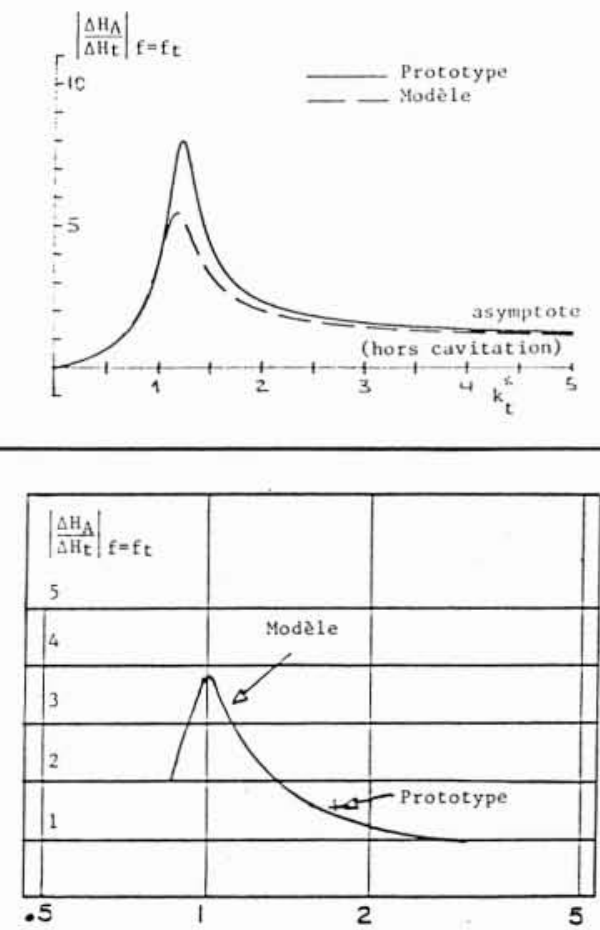

16.

COEFFICIENT DE CAVITATION RELATIF o/orm 


\section{Etude d'un cas réel (turbine nq 48). Comparaison modèle-prototype}

\section{IV.1. Présentation du modèle et du prototype}

$\begin{array}{llll}\text { Modèle } & \mathrm{H}=15 \mathrm{mCE} & \mathrm{n}=700 \mathrm{tr} / \mathrm{min} \mathrm{D}=0,338 \mathrm{~m} \\ \text { Amont } & \mathrm{L}=1,57 \mathrm{~m} & \mathrm{~S}=0,076 \mathrm{~m}^{2} \quad \mathrm{a}=1170 \mathrm{~m} / \mathrm{s} \\ \text { Aval } & \mathrm{L}=1,1 \mathrm{~m} & \mathrm{~S}=0,077 \mathrm{~m}^{2} & \mathrm{p} / \mathrm{o}=3,28 \\ \begin{array}{l}\text { Prototype } \\ \text { Amont }\end{array} & \mathrm{H}=125 \mathrm{~m} & \mathrm{n}=428 \mathrm{tr} / \mathrm{min} & \mathrm{D}=1,6 \mathrm{~m} \\ \quad & & & \\ \quad \text { Galerie } & \mathrm{L}=160 \mathrm{~m} & \mathrm{~S}=7,07 \mathrm{~m}^{2} & \mathrm{a}=900 \mathrm{~m} / \mathrm{s} \\ \quad \text { Forcée } & \mathrm{L}=283 \mathrm{~m} & \mathrm{~S}=4,52 \mathrm{~m}^{2} & \\ \text { Aval } & \mathrm{L}=20,6 \mathrm{~m} & \mathrm{~S}=5,76 \mathrm{~m}^{2} & \mathrm{p} / \mathrm{o}=3,28\end{array}$

La célérité de $900 \mathrm{~m} / \mathrm{s}$ correspond à une fréquence propre amont de $0,58 \mathrm{~Hz}$ mesurée sur site; la deuxième fréquence propre amont calculée vaut $1,72 \mathrm{~Hz}$.

\section{IV.2. Etude expérimentale sur modèle et proto- type}

L'expérimentation sur prototype a été effectuée pour trois valeurs du coefficient de cavitation $\sigma$ par modification du niveau aval; elle a été complétée sur modèle réduit, notamment par des mesures hors cavitation. L'ensemble des résultats est présenté sur les différentes figures données en Annexe. On notera les quelques points particuliers suivants :

- le point d'oscillation maximale se situe à une valeur de débit variant avec le coefficient de cavitation (figures 12, 13 et 14);

- les valeurs de $\sigma$ explorées sur prototype encadrent la résonance maximale (figure 2 );

- à faible charge, l'aspirateur est le siège d'un champ tournant (figure 12) qui évolue progressivement, pour les valeurs de $\sigma$ explorées sur prototype, vers une pulsation synchrone au régime d'oscillation maximale;

- l'évolution des oscillations maximales en fonction du débit d'aération diffère selon la valeur de $\sigma$ : pour $\sigma<\sigma_{m}$, on note une réduction progressive des oscillations; pour $\sigma>\sigma_{m}$, l'aération se traduit d'abord par une augmentation des oscillations avant qu'elle ne joue son rôle modérateur.

\section{IV.3. Etude dynamique théorique}

Le modèle mathématique exposé précédemment (§ III) est appliqué aussi bien sur modèle que sur prototype. (Les résultats du $\S$ III font également partie de cette application).

Nous supposons que l'excitation $\Delta H_{t}$ se transpose directement du modèle au prototype et que sa fréquence est indépendante de $\sigma$.

Les courbes de réponse obtenues (fig. 15) permettent de retrouver les valeurs d'amplitude mesurées à la résonance maximale, mais à condition d'ajuster les coefficients d'amortissement à des valeurs qui s'avèrent nettement différentes entre modèle et prototype (facteur 10). On voit donc que :

- les amortissements modélisés ne sont pas transposables. Ceci traduit vraisemblablement le fait que le phénomène d'amortissement est plus complexe que ce que nous avons modélisé.
- la résonance maximale se produit à souplesse de cavitation voisine, ce qui est en accord avec les résultats expérimentaux obtenus sur modèle et prototype.

\section{Remarques sur la transposition mo- dèle-prototype}

L'amplitude des oscillations de pression amont, de pression aval, de chute et de couple sur l'arbre n'est pas directement transposable du modèle au prototype. Théoriquement, le schéma suivant doit être appliqué, en tenant compte des fonctions de transfert de chacun des systèmes envisagés :

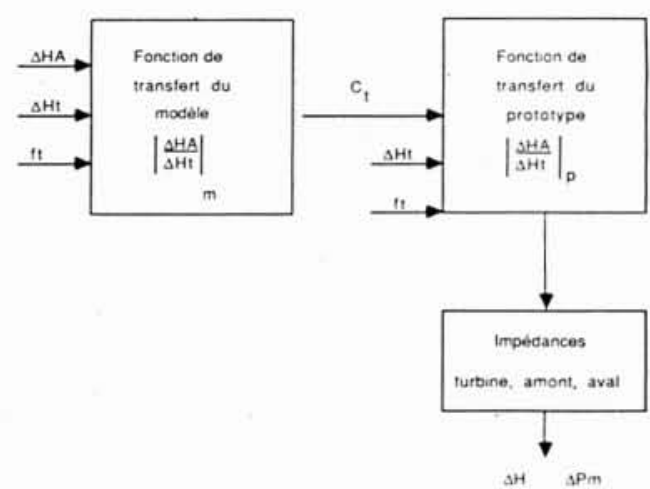

D'un point de vue pratique, la transposition n'est pas simple et des difficultés apparaissent : détermination des fonctions de transfert et des coefficients d'amortissement, influence de l'air dissous...

L'étude expérimentale faite sur une turbine $n_{q} 48$, qui fonctionne au voisinage du $\sigma$ de résonance maximale, montre que cette transposition de $C_{i}^{*}$ est possible moyennant certaines précautions. A cet égard, le choix de la référence du $\sigma$, hors similitude de Froude, a de l'importance: les essais sur modèle ont montré qu'elle devait être située à environ $0,7 \times D$ en-dessous de l'axe du distributeur.

Le schéma devient évidemment plus simple quand le circuit amont a un effet négligeable; tel est le cas quand le $\sigma$ de fonctionnement n'est pas trop proche du $\sigma$ de résonance maximale (Exemple fig. 16 avec turbine $n_{q} 84$ ).

\section{Conclusions}

La comparaison entre les essais effectués sur prototype et sur modèle réduit en similitude montre que les amplitudes et les fréquences des oscillations induites par la torche de cavitation de charges partielles ne sont pas directement comparables au voisinage de la résonance maximale. Par contre, on note une assez bonne transposition du point de fonctionnement et du coefficient de cavitation correspondant à la résonance maximale. 
La modélisation mathématique du système en vue d'étudier la transposition des oscillations induites par la torche de cavitation met en évidence les difficultés de prise en compte de lamortissement au voisinage de la résonance maximale. On note par contre que cette modélisation donne des résultats qualitatifs satisfaisants.

\section{Bibliographie}

[1] Dorfler P. - Modèle mathématique des oscillations excitées à charge partielle par la torche de cavitation dans les turbines Francis. Bulletin ESCHER WYSS 1/2 1980.

[2] Muciaccia F.F., Rossi G., ZanetTI V. - Study on model of the dynamic behaviour of hydraulic machine, $A I R H$, Striling 1984, article 3.10 .

[3] Ulith P., JaEger EU., Strscheletzky M. - Contribution to clarifying the inception of nonstationary flow pheno. mena in the drapht tube of high specific speed Francis turbine operating at part load. AIRH, symposium 1974, article III.4

[4] Nishi M., Matsunaga S. - Surging characteristics of conical and elbow-type draft tube, AIRH, Striling 1984 , article 3.6 .

[5] Campmas P. - Stabilité du régime de fonctionnement des turbines Francis. AIRH, Nice 1960, article B4.

[6] Dordain J.J., MaRcheTTI. - Matrice de transfert de systèmes hydrauliques. La recherche aérospatiale, $\mathrm{n}^{\circ}$ $1974-1$, p. $23-35$

[7] WYLIE EB. - Resonance in Pressurized Piping Systems. Transactions ASME - Journal Basic Engineering - Décembre 1965 .

[8] Chaudry MM. - Resonance in Pressurized Piping Systems. Journal of the hydraulics division ASCE - Septembre 1970 .

[9] Fanelli M. - Further consideration on the dynamic behaviour of hydraulic turbo-machinery. Water Power, Juin 1972, P. 208-222.

[10] Angelico G., Muclaccia F.F., Rossi G. - Part load behaviour of a turbine: study on a complete model of hydraulic power plant. Symposium IAHR-MONTREAL Septembre 1986.

[11] MARIA D. - Comportement dynamique d'une turbo-machine. Mesure indirecte sur modèle de turbine Francis. Note interne NEYRPIC du 12.07.1984.

\section{Notations}

$\Delta H_{t}:$ oscillation de pression excitatrice induite par la torche.

$\Delta H_{A}:$ composante synchrone de l'oscillation de pression dans l'aspirateur.

$\Delta H_{B}$ : oscillation de pression dans la bâche spirale.

$\Delta H$ : oscillation de pression de chute :

$\Delta H=\Delta H_{B}-\Delta H_{A}$

$f$ : fréquence $f=\omega / 2 \pi$

$f_{t}$ : fréquence d'excitation de la torche.

$f_{0}:$ fréquence de rotation de la roue.

$f_{p}$ : fréquence propre hydraulique.

$C_{t}$ : souplesse de cavitation.

$K_{t}:$ souplesse inverse $=\frac{1}{C_{t}}=-\frac{\Delta H A}{\Delta V}$

Paramètres réduits : $C_{i}^{*}=\frac{1}{K_{i}^{*}}=\frac{C t H}{D^{3}}$

$\Delta V:$ variation de volume de la torche.

$n \quad$ : vitesse de rotation.

$n_{q}=n \sqrt{Q} / H^{0,75}$ vitesse spécifique à l'optimum

0 : ouverture du distributeur.

$D$ : diamètre roue.

$H$ : chute nette.

$Q \quad$ : débit moyen turbiné.

$P_{e} \quad:$ puissance électrique.

$P_{m}$ : puissance mécanique.

$H_{r}^{*}$ : dépression relative sous la pointe de roue.

$\sigma:$ coefficient de cavitation de THOMA.

$\sigma_{r m}$ : coefficient cavitation de résonance maximale.

$L$ : longueur.

$S$ : section.

$Z$ : impédance.

$Z_{0}$ : impédance caractéristique $Z_{0}=a / g S$

$a \quad$ : célérité des ondes.

$C$ : coefficient de frottement à l'aval.

Indices :

$A$ : aspirateur.

$B$ : bâche.

$T$ : turbine.

$t$ : torche.

am : circuit amont.

av : circuit aval.

$\mathrm{rm}$ : résonance maximale.

Symbole:

$\Delta \quad$ : transformée de Fourrier : amplitude complexe de l'oscillation fonction de la fréquence. On considère dans cette note l'amplitude crête-à-crête (c.c.)
Adresses des auteurs

\author{
Monsieur A. Fritsch \\ EDF-DTG \\ Service essais hydrauliques \\ BP 41 Centre de tri \\ 38040 Grenoble Cedex \\ Tél. : 76.48 .84 .44
}

Monsieur D. Maria

Société Neyrpic

BP 75 Centre de tri

38041 Grenoble Cedex

Tél. : 76.39 .30 .00 
M. le Président. - Nous venons d'examiner dans la première partie de la matinée, sous les angles théoriques et expérimentaux, le problème des oscillations de pression des circuits et boucles d'essais. Nous passons dans la deuxième partie aux applications liées aux turbines hydrauliques.

Il va y avoir trois exposés, et dans ces exposés on va traiter des phénomènes liés au fonctionnement des turbines hydrauliques à des régimes qui s'écartent des paramètres optimaux du tracé. Dans ces conditions de fonctionnement, il se développe sous la roue un noyau cavitant appelé « torche ". Vous verrez le rôle fondamental de cette anomalie. Les deux premiers exposés vont traiter des problèmes particuliers du fonctionnement des turbines hydrauliques aux charges partielles. Le premier est axé sur une comparaison entre essais réalisés sur machines industrielles et essais sur modèles réduits correspondants avec modélisation théorique, avec un objectif plutôt qualitatif lié à la compréhenion des phénomènes. Ensuite nous verrons une modélisation beaucoup plus poussée au niveau de la torche.

... (présentation de l'exposé de MM. Fritsch et Maria)...

Merci Messieurs pour cette présentation. Je donne la parole aux auditeurs souhaitant poser des questions.

M. GRISON. - Pouvez-vous apporter des précisions sur le modèle mathématique employé pour le calcul de la turbine, en particulier utilisez-vous une modélisation quasi-statique ou instationnaire?

M. MARIA. - L'impédance de la turbine, on l'a constaté par ailleurs, se décompose en deux termes:

- une partie réelle qui en très basses fréquences nous intéresse. On peut considérer que c'est la part quasi-statique.

- et pour ce qui est de la partie imaginaire, on s'aperçoit que c'est un terme équivalent à un tronçon de conduite; cela se justifie par le fait que l'amenée de la turbine est une spirale assimilable à une portion de conduite.

L'impédance caractéristique évolue, mais en première approximation surtout dans les basses fréquences qui nous intéressent, cette approche est satisfaisante.

M. THIRRIOT. - Devant un aréopage de spécialistes, il est peut-être incongru de poser une question naive mais je vais le faire : "souplesse de cavitation ", le mot est joli mais qu'est-ce que cela représente?

M. MARIA. - C'est le rapport entre la variation de volume du noyau de vapeur (la variation de volume de la torche cavitante) et la variation de pression à ce niveau. En effet, au gré d'une variation de pression, la torche cavitante va voir ses caractéristiques de volume changer; globalement c'est un peu comme un coefficient de compressibilité.

M. FANELLI. - Vous avez montré le rôle important des caractéristiques des circuits amont et aval sur l'amplification, ainsi que l'influence fondamentale du $\sigma$ de cavitation. Vous avez aussi montré un cas de résonance avec le système électrique.

Dans des expériences réalisées chez nous, on a eu la possibilité de faire varier la fréquence propre fondamentale du système amont, et l'on a constaté effectivement une augmentation importante de l'amplification lorsqu'on amenait à la coïncidence cette fréquence et la fréquence de la torche. Par contre, dans notre modèle il n'était pas possible de faire varier le $\sigma$ de cavitation, et le $\sigma$ était tel qu'il n'y avait pas de poche de cavitation.
On a aussi fait varier, dans notre modèle, la fréquence d'oscillation torsionnelle de l'arbre de liaison machine hydraulique-machine électrique, mais on n'a constaté aucune amplification quand il y avait coïncidence entre cette fréquence torsionnelle et celle de la torche. Comment pouvez-vous expliquer cette absence de résonance?

M. MARIA. - Vos essais sont très intéressants.

Vous avez montré que les amplitudes augmentent dès qu'on se rapproche d'une fréquence propre du circuit amont. Cela a été fait hors cavitation, mais de toute façon le phénomène est le même.

Vous parlez aussi de faire varier la fréquence de torsion de l'arbre mécanique. Là le problème est différent.

On a une oscillation de pression à l'aval; il en résulte une oscillation de pression à l'amont. Ce qui crée l'oscillation de couple au niveau de l'arbre, c'est l'oscillation de chute, c'est-à-dire la différence entre l'amont et l'aval. Quand il y a coïncidence entre fréquence propre de torsion et fréquence de la torche, l'augmentation de l'oscillation de couple qui en résulte se répercute sur la vitesse de rotation et par voie de conséquence sur les pressions, mais probablement de manière relativement faible.

D'un autre point de vue, il faut considérer le comportement de l'amont par rapport à l'aval en fonction de la fréquence de la torche et de la fréquence propre amont. Avec un nœud de débit à l'entrée turbine, on tend vers une oscillation de chute et donc de couple nulle.

M. FABRI. - La torche est un phénomène essentiellement hydraulique qu'on ne devrait pas trouver dans les turbines à air. Or dans des essais effectués à l'ONERA sur une turbine à centripète, on a trouvé un phénomène un peu semblable, une sorte de " cavitation ", due aux basses pressions, apparait aux régimes partiels. On a pu mettre en évidence l'existence de vitesses tangentielles notables à la sortie de la turbine et le domaine des basses pressions s'est étendu du fait du tourbillon créé sur l'axe. Le phénomène a pu être maitrisé par la mise en place d'un corps solide, un cylindre, au niveau de l'axe, pour empêcher la formation de ce tourbillon.

Ce palliatif, efficace dans le cas d'une turbine à air, a-t-il été essayé dans une turbine hydraulique?

M. MARIA. - Oui c'est une solution très possible. Dans certains cas en effet, on a été amené à envisager de telles solutions (tube coaxial).

Le problème, c'est la tenue mécanique des supports. Théoriquement un obstacle permet d'égaliser en partie ce champ tournant, donc de faire baisser le degré d'excitation, mais en général on a un problème de tenue mécanique car les supports doivent offrir le minimum d'obstruction à l'écoulement.

M. LEFEBVRE. - Nous avons fait des essais sur un cyclindre coaxial mais nous $y$ avons renoncé pour des questions de rendement.

M. le Président. - Je vous propose de passer à l'exposé suivant. Nous venons de voir le rôle fondamental joué par la torche dans les excitations du circuit hydraulique d'une turbine. Il convient de représenter au mieux ce genre de phénomène pour l'approcher de façon satisfaisante sur le plan théorique.

C'est pourquoi M. Fanelli va présenter un exposé sur la « Modélisation mathématique de la torche à l'aspiration d'une turbine Francis fonctionnant à charge partielle ". 


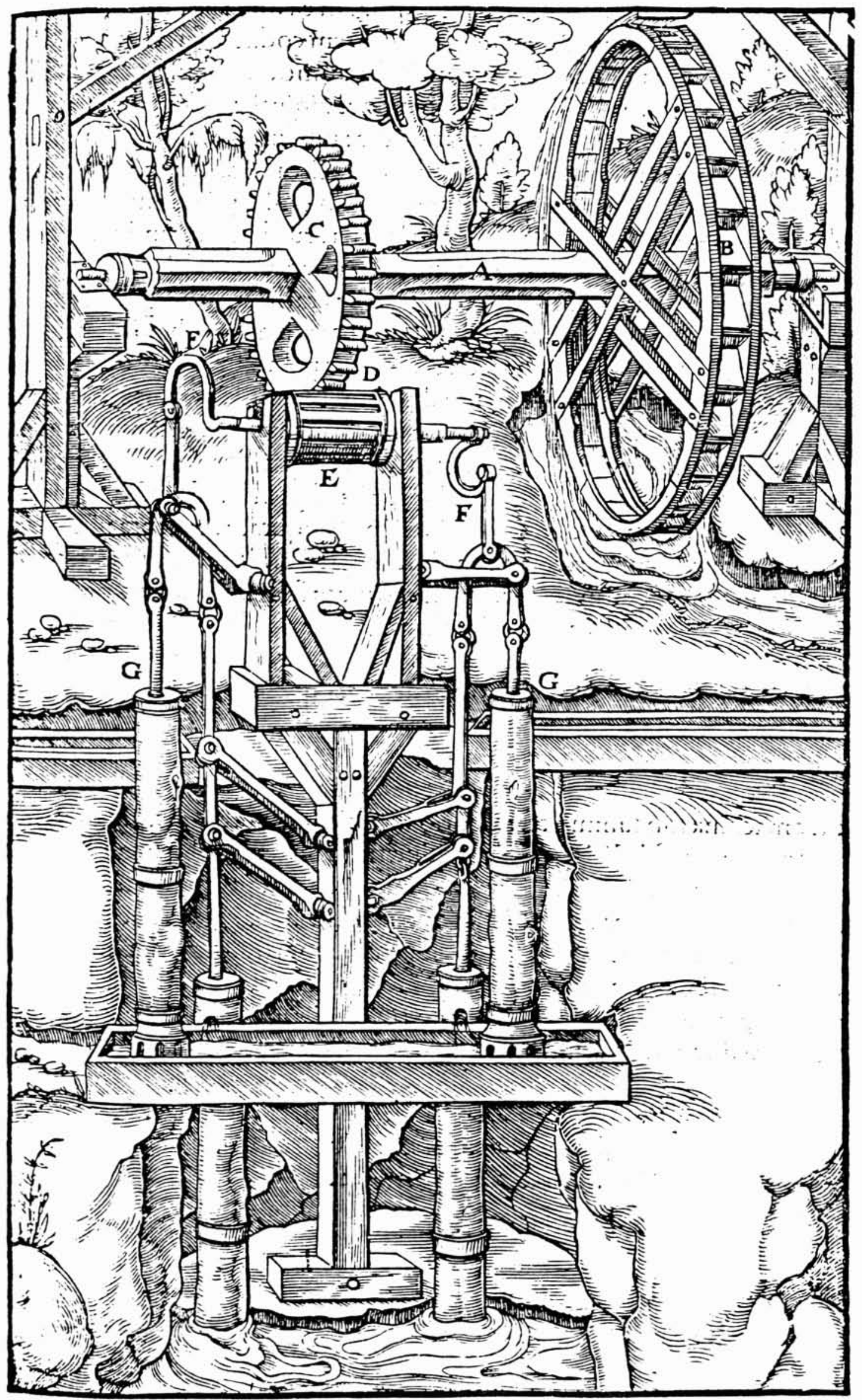

